Bundesgesundheitsbl - Gesundheitsforsch Gesundheitsschutz 2004 • 47:896-907 DOI 10.1007/s00103-004-0895-3

c) Springer Medizin Verlag 2004

\author{
U. Marcus \\ Robert Koch-Institut, Berlin
}

\title{
11. Retroviruskonferenz in San Francisco, 8.-11. Februar 2004
}

\section{Teil II: Therapiestrategien, Therapieneben- wirkungen, virale Resistenzentwicklung und Resistenzübertragung, HCV-Koinfektion}

Nach 3 strukturierten Behandlungsunterbrechungen wurde die Therapie schließlich nach 84 Wochen abgesetzt und der weitere Verlauf beobachtet. Beendet wurde die Studie von 26 Patienten. Bei mehr als der Hälfte musste Hydroxyurea wegen Toxizität vorzeitig abgesetzt werden. In der 108. Woche, also 24 Wochen nach Absetzen der Therapie, lag die Viruslast bei 7 von 26 Teilnehmern unter 1.000 Viruskopien/ml, bei den übrigen deutlich darüber. Bei 3 Patienten hatten sich PI-Resistenzmutationen entwickelt [Hoen et al. Abstr. 395]. Im Rahmen dieser Studie wurde bei 22 Teilnehmern auch die CD4- und CD8-vermittelte HIV-Immunantwort untersucht. Obwohl die verwendeten Testmethoden eine Stärkung der CD8- und CD4-vermittelten Immunantwort nahe legen, gelang der Nachweis nicht, dass diese auch zu einer wirksameren Kontrolle der Plasmaviruslast führt [Lacabaratz-Porret et al. Abstr. 396].

In einer Analyse von 3 prospektiven Kohorten zur Therapie der HIV-Primärinfektion wurde untersucht, ob der Zeitpunkt des Behandlungsbeginns (vor oder während der Serokonversion vs. innerhalb von 6 Monaten nach Serokonversion) einen Einfluss auf den längerfristigen Ver- lauf der Viruslast oder der T-Helferzellzahl hat. Ausgewertet wurden 117 Patienten, die die Behandlung nach der Serokonversion begonnen hatten, und 51 Patienten, die die Behandlung bereits vor oder während der Serokonversion begonnen hatten. Innerhalb der ersten 3 Jahre war zwischen den beiden Gruppen im Hinblick auf die Viruslast und die T-Helferzellzahl kein signifikanter Unterschied feststellbar [Voirin et al. Abstr. 23].

Aus Frankreich wurde über einen Vergleich zwischen einer Gruppe von $58 \mathrm{~Pa}$ tienten, die eine Behandlung im Rahmen der HIV-Primärinfektion begonnen und später (nach ca. 24 Monaten) wieder abgebrochen hatten, und einer Gruppe von 116 Serokonvertern, die nicht behandelt worden waren, berichtet. 3 Jahre nach der Infektion lag die Viruslast bei den Frühtherapierten im Mittel bei 3,75 log, bei den Spontanverläufen ohne Behandlung bei 3,94 log - kein besonders überzeugender Unterschied [Desquilbet et al. Abstr. 397].

Noch nicht ganz auszuschließen ist, dass durch spezielle Substanzen, wie z. B. Mycophenolat Mofetil (eine Substanz, die die Zellaktivierung reduziert), das Behandlungsergebnis einer Frühtherapie optimiert werden kann. In einer italienisch- 
schweizerischen Pilotstudie mit 14 Teilnehmern, die während der Unterbrechung der antiretroviralen Therapie Mycophenol eingenommen hatten, konnten 11 Patienten eine effektive Kontrolle der Plasmaviruslast über einen Zeitraum von 48 Wochen aufrechterhalten [Rizzardi et al. Abstr. 400].

\section{Therapeutische Immunisierung - kein Erfolg in Sicht}

Die Ursache für das Ausbleiben eines Erfolgs der Strategie der strukturierten Therapieunterbrechungen scheint in der Ausschaltung HIV-spezifischer Immunzellen durch die persistierende bzw. nach Therapieunterbrechung wieder aufflammende Virusreplikation zu liegen. Dieses Hindernis könnte durch eine therapeutische Immunisierung unter dem Schutz einer gleichzeitigen hochaktiven antiretroviralen Therapie (HAART) umgangen werden. In San Francisco wurden mehrere Studien vorgestellt, in denen mit verschiedenen Immunogenen bei HIV-Infizierten unter einer HAART versucht wurde, die HIV-spezifische Immunität zu stärken. Das Gelingen dieser Strategie wurde durch Unterbrechung der antiretroviralen Therapie getestet. In der randomisierten und kontrollierten französischen ANRS095-Studie wurden 43 frisch mit HIV infizierte Patienten in 3 Vergleichsgruppen behandelt: Gruppe 1 wurde mit antiretroviraler Kombinationstherapie (HAART) behandelt, Gruppe 2 mit HAART und 5 Zyklen subkutanem Interleukin 2 und Gruppe 3 mit HAART plus 4 intramuskulären Injektionen eines rekombinanten Vogelpockenvirus mit HIV-Sequenzen und einer Mischung aus rekombinanten HIV-Lipopeptiden, gefolgt von 3 Zyklen Interleukin 2. Bei 39 Teilnehmern wurde die Therapie nach 40 Wochen abgesetzt. In Woche 52 wiesen noch 29\% der Teilnehmer der Gruppe 1 eine Viruslast von $<10.000$ Kopien auf, in der Gruppe 2 waren es $43 \%$ und $47 \%$ in der Gruppe 3 . Die mittlere Zeitspanne bis zu einem virologischen Versagen lag in der Gruppe 1 bei 42 Tagen, in der Gruppe 2 bei 58 Tagen und in der Gruppe 3 bei 63 Tagen. Angesichts der kleinen Fallzahlen waren diese Unterschiede statistisch nicht signifikant [Goujard et al. Abstr. 285].
In der internationalen randomisierten, placebokontrollierten QUEST-Studie erhielten 79 frisch Infizierte, die seit mindestens 1,5 Jahren erfolgreich mit einer HAART behandelt worden waren, entweder Placebo, eine Immunisierung mit einem rekombinanten HIV-Vogelpockenvirus oder eine Immunisierung mit dem rekombinanten Virus plus Remune (eine Präparation von inaktiviertem HIV, bei dem das Hüllprotein weitgehend fehlt). Nach der Immunisierung wurden eine analytische Therapieunterbrechung für 24 Wochen vorgenommen und Viruslast und T-Helferzellzahl bestimmt. Es konnte kein Unterschied zwischen der Placebo- und den beiden Immunisierungsgruppen festgestellt werden [Kinloch et al. Abstr. 168]. Ähnlich erfolglos war ein Immunisierungsversuch in Australien, bei dem zur Immunisierung ein anderes rekombinantes HIV-Vogelpockenvirus mit oder ohne Interferon- $\gamma$ verwendet wurde. Tendenziell waren in dieser Studie die Ergebnisse in der Impfstoff-Interferon- $\gamma$-Gruppe zwar etwas besser, aber auch hier waren die Unterschiede statistisch nicht signifikant [Cooper et al. Abstr. 169].

Diese Ergebnisse zeigen, dass zumindest mit den bisher verwendeten Impfstoffen und Impfschemata die erhoffte Stärkung der HIV-spezifischen Immunität nicht erreicht werden konnte.

In der hinsichtlich eines Schutzeffektes erfolglosen amerikanisch-europäischen Impfstoffwirksamkeitsstudie mit dem rekombinanten Subtyp-B-Hüllprotein wurde untersucht, ob bei den Impfversagern ein Einfluss auf die Höhe und den Verlauf der Viruslast nach Infektion feststellbar war. Dies war nicht der Fall [Gilbert et al. Abstr. 283]. Bei Teilnehmern von französischen Phase-I-Impfstudien wurden im HIV-Elisatest die Zahl und die Dauer einer Seropositivität nachuntersucht. Bei 41\% der Impfstudienteilnehmer fiel der Elisatest im Mittel noch 7 Jahre nach Immunisierung positiv aus. Angesichts potenziell negativer sozialer Konsequenzen einer Reaktivität in HIV-Screening-Testen ist es wichtig, dass die Impfung und die Möglichkeit eines positiven Elisatestes auf einem speziellen Impfausweis vermerkt und die Teilnehmer über diese Möglichkeit aufgeklärt werden [Silbermann et al. Abstr. 289].
Im SIVmac239/Rhesusaffenmodell wurde geprüft, ob eine Alloimmunisierung gegen $\mathrm{MHC}$-Antigene $\mathrm{zu}$ einem Impfschutz gegen SIV führen kann. Obwohl eine kräftige humorale und zelluläre Immunantwort gegen MHC-Fremdantigene erzielt werden konnte, war diese aber nicht in der Lage, einen Schutz nach intravenöser oder intrarektaler Virusinokulation zu gewähren [Guan et al. Abstr. 276].

Die Mechanismen, die bei Infektionen mit den Modellviren SIVmac239 und der rekombinanten Viruschimäre SHIV $_{\text {DH12R }}$ zur Ausbildung eines Immundefektes führen, wurden von einer amerikanischen Arbeitsgruppe analysiert. Während SIV hierfür einen Zeitraum von einem bis 3 Jahren benötigt, verursacht SHIV bereits innerhalb weniger Wochen einen irreversiblen T-Helferzellverlust. Obwohl SHIV daher als viel aggressiver erscheint, lässt sich ein Impfschutz gegen dieses Modellvirus viel leichter erzielen als gegen SIV. Diese Tatsache spricht für Unterschiede in den pathogenetischen Mechanismen der beiden Viren. Tatsächlich verwendet die SHIV-Chimäre für die Infektion ausschließlich den CXCR4-Rezeptor, während SIV CCR5 benutzt. Nach einer SHIV-Infektion kommt es zu einem massiven Verlust CXCR4-positiver naiver CD4-Zellen, während nach einer SIV-Infektion selektiv CCR5-positive Gedächtniszellen verloren gehen. Die Höhe des CD4-Zellverlustes ist von der Infektionsdosis abhängig, bei SIV ist dies nicht der Fall. Durch eine medikamentöse Unterdrückung der SHIV-Replikation innerhalb der ersten 2 Wochen nach Infektion kann ein symptomfreier Langzeitverlauf der Infektion induziert werden, während eine vergleichbare Frühtherapie nach SIV-Infektion keinen krankheitsverhindernden Effekt hat. Diese Ergebnisse lassen den Schluss zu, dass die Erfolge von Impfstudien im SHIV-Modellsystem wahrscheinlich nur eine geringe Relevanz für die HIV-Infektion aufweisen [Igarashi et al. Abstr. 127].

\section{Therapiestudien}

In der Bristol-Myers Squibb-Studie A1424-045 wurde die Wirksamkeit von 
geboostetem $^{1}$ Atazanavir (300/100 mg 1-mal/Tag) mit der Wirksamkeit von Lopinavir/rit (400/100 mg 2-mal/Tag) und einem Doppel-PI- (Proteaseinhibitor-)Arm, bestehend aus Atazanavir und Saquinavir (400/1.200 mg 1-mal/Tag), jeweils in Kombination mit Tenofovir und einem anderen nukleosidischen Reverse Transkriptase-Inhibitor (NRTI), verglichen. Die Studienteilnehmer waren alle vorbehandelt und hatten bereits ein Versagen von mindestens 2 Behandlungsregimen hinter sich. Gemessen an Viruslast und T-Helferzellzahl schnitten die beiden geboosteten PI-Arme gleich gut ab, während der Doppel-PIArm eine geringere Wirksamkeit aufwies. Dies mag mit einer Absenkung der Atazanavir-Plasmaspiegel durch Tenofovir und damit auch einer zu geringen Boosterwirkung von Atazanavir auf den Saquinavirspiegel zusammenhängen (Plasmaspiegelmessungen wurden noch nicht ausgewertet) [DeJesus et al. Abstr. 547].

In einer Studie mit knapp 200 Therapie-naiven Teilnehmern wurde die einmal tägliche Gabe von Lopinavir/rit (800/200 mg) mit der 2-mal täglichen Gabe (400/100 mg), jeweils in Kombination mit Emtricitabin und Tenofovir (beide einmal täglich) verglichen. Bezüglich der virologischen und immunologischen Wirksamkeit ließ sich nach 48 Wochen Behandlung kein Unterschied ausmachen. Die Nebenwirkung Durchfall trat bei einmal täglicher Lopinavir-Gabe häufiger auf (13\% vs. $5 \%)$, sonst gab es keine nennenswerten Unterschiede im Hinblick auf die Verträglichkeit [Gathe et al. Abstr. 570].

Nach dem schlechten Abschneiden bestimmter Tripelnukleosidanaloga-Kombinationen $(\mathrm{ABC}+3 \mathrm{TC}+\mathrm{TNF})$ wurde spekuliert, ob Abacavir bei einmal täglicher Gabe zu niedrige intrazelluläre Wirkspiegel aufweist und als Folge ein Therapieversa-

\footnotetext{
${ }^{1}$ Unter dem Begriff Boosterung versteht man die Hemmung des Enzymsystems in der Leber, das für den Abbau verschiedener Substanzen, u. a. auch von Proteaseinhibitoren und nichtnukleosidischen Reverse Transkriptase-Inhibitoren (NNRTI) verantwortlich ist. Dadurch wird der Wirkspiegel der Medikamente über längere Zeit auf einem höheren Niveau gehalten. Die stärkste bekannte Hemmsubstanz des Enzymsystems ist der Proteaseinhibitor Ritonavir, der inzwischen fast ausschließlich in subtherapeutischer Dosierung zur Boosterung anderer Proteaseinhibitoren eingesetzt wird.
}

gen auftreten könnte. In einer GlaxoSmithKline-Studie (CNA30021) wurde die einmal tägliche Abacavir-Dosierung mit einer 2-mal täglichen, jeweils kombiniert mit Lamivudin und Efavirenz, verglichen. Nach 48 Wochen Behandlung waren hinsichtlich der virologischen Wirksamkeit und Resistenzentwicklung keine Unterschiede zwischen den beiden Behandlungsarmen auszumachen. Ein virologisches Versagen wurde in den beiden Studienarmen bei 10 bzw. 8\% der Teilnehmer registriert [Craig et al. Abstr. 551]. Das Ergebnis spricht dafür, dass bei den richtigen Kombinationspartnern eine einmal tägliche AbacavirGabe möglich ist.

Ein unerwarteter Lymphozytenabfall trotz virologisch erfolgreicher Therapie wird bei Patienten beschrieben, die im Rahmen von Kombinationsregimen mit Didanosin und Tenofovir in Standarddosierung behandelt werden. In dieser Kombination kommt es zu einer Wechselwirkung zwischen Tenofovir und Didanosin, die in einem erhöhten Didanosin-Plasmaspiegel resultiert. Bei Reduktion der Didanosin-Dosis von $400 \mathrm{mg} / \mathrm{Tag}$ (bei einem Gewicht von $>60 \mathrm{~kg}$ ) auf $250 \mathrm{mg} / \mathrm{Tag}$ werden dieselben Plasmaspiegel wie mit der 400-mgDosis ohne Tenofovir erreicht. Ob der Lymphozytenabfall allein auf toxisch erhöhte Didanosin-Spiegel zurückzuführen ist oder ob noch andere Mechanismen eine Rolle spielen, ist noch nicht abschließend geklärt [Negredo et al. Abstr. 749].

In der europäischen COLATE-Studie wurde überprüft, ob die Weiterbehandlung mit Lamivudin nach dem Versagen eines Lamivudin-haltigen Regimes einen klinischen Nutzen besitzt (über die Beibehaltung der M184V-Mutation). Bei 124 nach 48 Wochen auswertbaren Patienten war kein statistisch signifikanter Vorteil der Weiterbehandlung nachweisbar, wohl aber ein schwacher Trend hin zu einer besseren virologischen Wirksamkeit. Möglicherweise ist ein positiver Effekt der Weiterbehandlung zwar vorhanden, ist aber zu gering, um bei der relativ geringen Teilnehmerzahl ein statistisch signifikantes Ergebnis zu zeigen [Dragsted et al. Abstr. 549]. In einer Analyse von Prädiktoren des Therapieversagens in der $2 \mathrm{NN}$-Studie (Nevirapin vs. Efavirenz+2NRTI) kristallisieren sich eine sehr niedrige AusgangsCD4-Zahl (<25 CD4-Zellen/ $/$ l) und eine hohe Viruslast (>100.000 Kopien/ml) als Risikofaktoren für ein Therapieversagen heraus [van Leth et al. Abstr. 550].

Eine englische Pharmakokinetikstudie untersuchte, wie lange nach dem Absetzen von Efavirenz therapeutische Plasmaspiegel nachweisbar bleiben. Bei 4/7 Patienten waren ausreichende Plasmaspiegel noch nach 7 Tagen nachweisbar, bei 3/7 Patienten noch nach 14 Tagen und bei 2/7 Patienten sogar noch 21 Tage nach Absetzen. Zum Schutz vor einer Resistenzentwicklung durch eine Quasi-Monotherapie mit Efavirenz (bzw. Nevirapin) ist daher zu empfehlen, andere Kombinationspartner entweder mindestens eine Woche nach Absetzen weiterlaufen zu lassen oder Efavirenz zunächst gegen eine Substanz mit kürzerer Halbwertzeit auszutauschen und diese Therapie erst 2-3 Wochen später abzusetzen [Taylor et al. Abstr. 131]. In einer Analyse bei 35 Teilnehmern von Studien mit strukturierten Therapieunterbrechungen wird das erhöhte Risiko einer Resistenzentwicklung durch gleichzeitiges Absetzen aller Medikamente bei NNRTI-haltigen Therapieregimen bestätigt. Nach Unterbrechung von NNRTI-Regimen betrug das Resistenzentwicklungsrisiko 44\%, nach Unterbrechung einer PI-haltigen Kombination hingegen nur 3\% [Arnedo-Valero et al. Abstr. 668].

In einer italienischen Studie wurde ebenfalls eine erhöhte Rate von Resistenzentwicklungen nach Therapieunterbrechung registriert. Als besonders gefährdet erwiesen sich Patienten, die bereits vor der Unterbrechung Resistenzen aufwiesen [Palmisano et al. Abstr. 552]. In einer nachträglichen Analyse von Prädiktoren des Therapieversagens in der ACTG-388-Studie [Indinavir (IDV) vs. Efavirenz (EFV)+IDV vs. Nelfinavir (NFV)+IDV jeweils kombiniert mit Zidovudin (ZDV)+Lamivudin (3TC)] waren Therapieunterbrechungen ebenfalls mit einem Therapieversagen assoziiert. Weitere Risikofaktoren waren jüngeres Alter und Zugehörigkeit zu einer nicht-weißen Bevölkerungsgruppe. Kurzfristige vorübergehende Viruslastanstiege auf $>50 \mathrm{Kopien} / \mathrm{ml}$ und kurz zuvor aufgetretene toxische Nebenwirkungen scheinen dagegen keinen Einfluss auf das Therapieversagen gehabt zu haben [Ribaudo et al. Abstr. 553].

Der Einfluss der ethnischen Zugehörigkeit auf Resistenzentwicklung und The- 
Tabelle 1

FORTE-Studie - Wirksamkeit und Nebenwirkungen (NW)

\begin{tabular}{|llll}
\hline & $\begin{array}{l}\text { Standard- } \\
\text { therapie }\end{array}$ & $\begin{array}{l}\text { Induktions-I } \\
\text { Erhaltungstherapie }\end{array}$ & $\begin{array}{l}\text { Signifikanz- } \\
\text { level }\end{array}$ \\
$\begin{array}{l}\text { Virologisches Versagen } \\
\text { nach 32 Wochen }\end{array}$ & $43 \%$ & $18 \%$ & $\mathrm{p}=0,002$ \\
\hline Viruslast (VL) $<50$ in Woche 48 & $65 \%$ & $81 \%$ & $\mathrm{p}=0,07$ \\
\hline Schwere NW & 13 & 17 & $\mathrm{~ns}$ \\
\hline NW Grad 3 oder 4 & 15 & 21 & $\mathrm{~ns}$ \\
\hline $\begin{array}{l}\text { Wechsel eines Medikaments } \\
\text { wegen NW }\end{array}$ & 24 & 25 & $\mathrm{~ns}$ \\
\hline Progression zu AIDS/Tod & 4 & 7 & $\mathrm{~ns}$ \\
\hline
\end{tabular}

rapieversagen von NNRTI enthaltenden Kombinationen hat möglicherweise seine Ursache in einer verlangsamten Verstoffwechselung von Efavirenz aufgrund genetisch bedingter Variation der Cytochromzusammensetzung in der Leber. Bei Absetzen von Efavirenz führt dies zu einem langsameren Abfall der Plasmaspiegel, was eine Resistenzentwicklung begünstigen könnte. Außerdem ist das entsprechende Cytochrommuster mit einer erhöhten Rate an ZNS-Nebenwirkungen durch Efavirenz verbunden [Taylor et al. Abstr. 131; Ribaudo et al. Abstr. 132; Haas et al. Abstr. 133].

Zahlreiche Studien beschäftigten sich mit verschiedenen Formen der Therapieintensivierung: Die englische FORTEStudie untersuchte, ob eine Induktionstherapie mit 4 Substanzen (2 NRTI+1 NNRTI+1 PI), gefolgt von einer Erhaltungstherapie mit 3 Substanzen (2 NRTI+1 NNR$\mathrm{TI}$ ), weniger Therapieversager zur Folge hat als eine Standardtherapie mit 2 NRTI+1 NNRTI. Die anfängliche Induktionstherapie erfolgte über 24-32 Wochen. An der Studie nahmen 122 Patienten teil, die im Mittel über 81 Wochen verfolgt werden konnten. Die intensivierte Induktionstherapie reduzierte das Risiko eines frühen virologischen Versagens, was aber durch eine tendenziell etwas höhere Nebenwirkungsrate (Unterschied statistisch nicht signifikant) erkauft wurde (- Tabelle 1) [Williams et al. Abstr. 564].

Trotz virologisch erfolgreicher HAART lassen sich bei antiretroviral Behandelten in der Regel noch eine erhöhte Immunaktivierung und andere immunologische Auffälligkeiten nachweisen. In einer kleinen amerikanischen Studie mit 17 Patien- ten wurde das laufende, prinzipiell erfolgreiche Behandlungsregime [Viruslast (VL) $<400 \mathrm{Kopien} / \mathrm{ml}$ ] durch Austausch des Proteaseinhibitors gegen Lopinavir/rit und zusätzliche Tenofovir-Gabe intensiviert, um zu prüfen, ob dadurch die Immunaktivierung weiter verringert, andere Immunparameter günstig beeinflusst und das latente Virusreservoir reduziert werden kann. Eine wesentliche Verbesserung der fraglichen Parameter konnte durch die Intensivierung jedoch nicht erreicht werden [Smith et al. Abstr. 228]. In der ACTG-372A-Studie wurde in ähnlicher Weise eine bis dahin erfolgreiche Therapie (VL $<500$ Kopien/ml) mit IDV+2 NRTI durch zusätzliche Gabe von Abacavir intensiviert, um ein virologisches Versagen weiter hinauszuzögern. Dieses Ziel wurde ebenfalls nicht erreicht: virologisches Versagen, Frequenz intermittierender Virämien, Restvirämie unter 50 Kopien/ml, CD4-Zellzahl und Resistenzprofil bei virologischem Versagen wurden durch die Intensivierung nicht spürbar beeinflusst [Hammer et al. Abstr. 56].

\section{Immunologische Effekte bei Behandlungsunterbrechungen}

Der Verlauf der T-Helferzellzahlen bei Therapieunterbrechungen wurde bei 195 Patienten aus 3 Studien untersucht. Bei allen Teilnehmern umfasste die Therapiepause mindestens 4 Wochen (bei 94 von ihnen sogar 24 Wochen oder länger). Der - unter Therapiepause zu erwartende - T-Helferzellabfall verläuft, ebenso wie der Anstieg bei Aufnahme einer antiretroviralen Therapie, 2-phasig: in den ersten 4 Wochen einer Unterbrechung sinkt die CD4-
Zellzahl im Mittel um 35 Zellen/ $\mu$ l pro Woche, in den darauf folgenden Wochen im Mittel nur noch um 4 Zellen/Woche [Fagard et al. Abstr. 568].

Die zelluläre Immunantwort gegen opportunistische Erreger und gegen HIV wurde bei 12 Patienten engmaschig untersucht, die die antiretrovirale Therapie auf eigenen Wunsch unterbrachen. Bei allen Patienten stieg die Viruslast innerhalb von 6 Wochen nach Absetzen der Therapie auf Werte von über 100.000 Kopien/ $\mathrm{ml}$ an. Parallel zum Viruslastanstieg wurde die lymphoproliferative Antwort auf opportunistische Erreger schwächer, normalisierte sich aber, sobald sich die HIV-Spiegel wieder auf ein Plateau eingependelt hatten. Die lymphoproliferative Antwort gegen HIV-Antigene wurde durch das Absetzen der Behandlung nicht beeinflusst. Die Stärke der HIV-spezifischen zellulären Immunität war mit der Höhe des Viruslastgipfels, der nach der Therapieunterbrechung erreicht wurde, assoziiert [Schrier et al. Abstr. 237].

In einer weiteren Studie, in der immunologische Parameter bei 14 Teilnehmern untersucht wurden, die eine virologisch erfolgreiche ART unterbrachen, zeigte die Stärke der HIV-spezifischen lymphoproliferativen CD4-Zellantwort ebenfalls keinen Einfluss auf die Höhe des Viruslastanstiegs während der Therapiepause. Bei der Hälfte der Patienten wurden unter Therapie vorübergehende Phasen einer geringgradigen Virämie (sog. Virus-Blips) beobachtet. Obwohl bei diesen Patienten unter Therapie die lymphoproliferative Antwort gegen HIV stärker ausfiel als bei den Patienten ohne Blips, war bei ihnen der Viruslastanstieg in der Therapiepause stärker [Papasavvas et al. Abstr. 233].

In der italienischen BASTA-Studie wurde bei 114 Patienten unter virologisch und immunologisch erfolgreicher HAART die Therapie unterbrochen. Primärer Endpunkt der Studie war ein Abfall der T-Helferzellzahl unter einen Wert von 400 Zellen/ $\mu$ l. Als einziger unabhängiger Vorhersageparameter für das Erreichen dieses Endpunktes erwies sich die tiefste bei dem jeweiligen Patienten zuvor erreichte T-Helferzellzahl, d. h., bei den meisten Patienten, die vor Behandlungsbeginn weniger als $400 \mathrm{~T}-\mathrm{Hel}-$ ferzellen aufwiesen, fiel die Zellzahl innerhalb des 18-monatigen Beobachtungszeit- 
raumes nach Therapieunterbrechung wieder ab, während der Wert bei allen Patienten mit Ausgangswerten von >500 Zellen/ $\mu$ l oberhalb der Grenze von 400 Zellzahlen/ $\mu \mathrm{l}$ blieb [Maggiolo et al. Abstr. 238].

Es wurden auch die Ergebnisse der ACTG-A5068-Studie, einer kontrollierten Studie zur Wirksamkeit einer 16-wöchigen Therapiepause vor Beginn einer Salvage-Therapie, vorgestellt. Untersucht wurde, ob die in der Behandlungspause erfolgende Reversion der resistenten Viruspopulation zurück zum Viruswildtyp die Antwort auf das Salvage-Regime verbessert. Im Vergleichsarm wurde ohne Therapiepause auf das laut Resistenztestung und Behandlungsvorgeschichte aussichtsreichste Behandlungsregime umgestellt. Die Patienten im Studienarm mit Therapiepause schnitten in keinem Parameter günstiger ab als die Patienten im Therapiearm ohne Therapieunterbrechung. Bei Patienten, bei denen es zu einer Reversion der Viruspopulation zum Wildtyp gekommen war und die unter dem Salvage-Regime virologisch versagten, wurde wieder dieselbe multiresistente Viruspopulation selektiert wie vor der Therapiepause. Virus mit Resistenzen gegen 3 Medikamentenklassen wurde auch dann selektioniert, wenn das Salvage-Regime nur aus einer oder 2 Medikamentenklassen bestand [Benson et al. Abstr. 58].

Über den klinischen Nutzen von Medikamentenspiegelbestimmungen berichtete eine spanische Arbeitsgruppe. Bei 143 Patienten wurden Spiegel von Proteaseinhibitoren und NNRTIs bestimmt. Anlässe hierfür waren Verdacht auf Medikamententoxizität (59\%), unerwartetes virologisches Versagen (39\%) und mögliche Medikamenteninteraktionen (2\%). Erhöhte Spiegel wurden bei 37\% der Patienten mit Verdacht auf toxische Effekte, zu niedrige Spiegel bei $42 \%$ der Patienten mit Therapieversagen gemessen. Bei 20 Patienten wurde daraufhin die Dosierung verändert oder die Therapie umgestellt, bei 16 Patienten wurde auf diese Weise das jeweilige klinische Ziel erreicht [Rendon et al. Abstr. 567].

\section{Klinische Wirksamkeit der Kombinationstherapien}

Die zunehmende Erfahrung im Einsatz antiretroviraler Kombinationstherapien

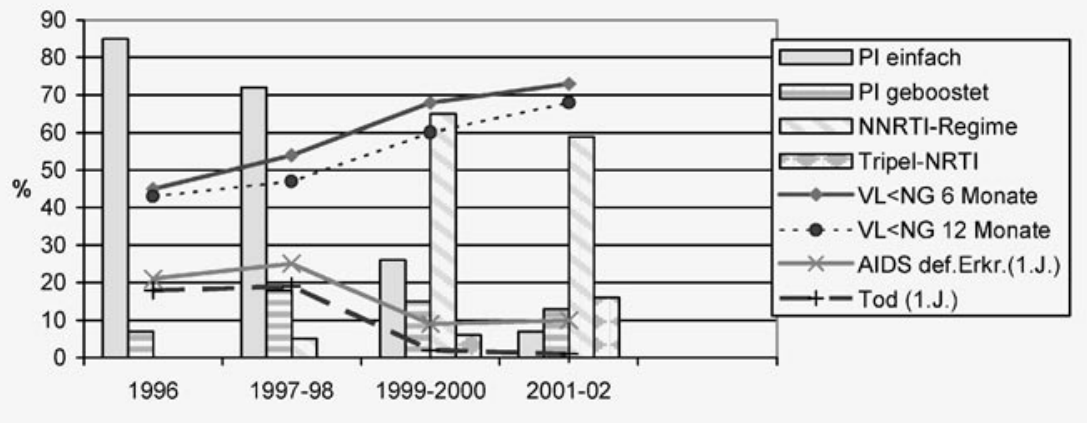

Abb. $1 \triangle$ Zeitliche Korrelation des Einsatzes unterschiedlicher Kombinationsregime mit virologischem Therapieerfolg und Auftreten AIDS-definierender Erkrankungen bzw. Tod. PI Protease-Inhibitor, NG Nachweisgrenze, NNRTI nicht nukleosidische Reverse Transkriptase-Inhibitoren, VL Viruslast

und die Verwendung wirksamerer Medikamentenkombinationen wie geboosteter PIs schlägt sich in einer Reduktion der Krankheitsprogressionsraten, der Todesfälle und einer verbesserten virologischen Wirksamkeit der Therapien im Vergleich zu den ersten Jahren nach Etablierung der HAART nieder. Eindrucksvoll lassen sich die Fortschritte durch einen entsprechenden Vergleich der zu verschiedenen Zeitpunkten behandelten Patientenkohorten demonstrieren (• Abb. 1) [Moore et al. Abstr. 558].

Die zunehmende Wirksamkeit der antiretroviralen Behandlung wird auch durch eine Analyse von Todesursachen und Erkrankungen in der HOPS-Kohorte $(n=5.561$ Teilnehmer $)$ belegt. Die Sterberate sank von 6,3/100 Personenjahre im Jahr 1996 auf 2,2/100 Personenjahre im Jahre 2002. Der stärkste Rückgang fand zwischen 1996 und 1998 statt. Die Erkrankungswahrscheinlichkeit für opportunistische Infektionen ging ebenfalls von 23/100 Personenjahre (1996) auf 6/100 Personenjahre (2002) zurück. Der Anteil der mit HAART behandelten Patienten stieg von 48\% im Jahr 1996 auf $80 \%$ im Jahr 2002. Bei den im gesamten Zeitraum von 1996-2002 registrierten 473 Todesfällen stieg der Anteil nichtopportunistischer Erkrankungen als Todesursache von 45,7\% im Jahr 1996 auf 71,7\% im Jahr 2002. Die am nächsten zum Todeszeitpunkt bestimmte T-Helferzellzahl stieg im Mittel von 66 Zellen/ $\mu \mathrm{lim}$ Jahr 1996 auf 148 Zellen/ $\mu \mathrm{lim}$ Jahr 2002. Die häufigsten nicht-opportunistischen zum Tode führenden Erkrankungen betrafen die Leber (36\%), die Lungen $(23 \%)$, das Herz-Kreislauf-System
(17\%) und die Niere (10\%) [Palella et al. Abstr. 872].

In einer Analyse der Todesursachen von HIV-infizierten Personen aus New York und San Francisco zwischen 1987 und 1999 waren 88,3\% der Todesfälle auf die HIV-Infektion zurückzuführen. Der Anteil nicht HIV-bedingter Todesursachen fiel zunächst von 10,6\% im Jahr 1987 auf 8,4\% im Jahr 1995, um dann bis 1999 wieder auf 22,9\% anzusteigen. Häufigste andere Todesursache waren bei dieser Untersuchung Herz-Kreislauf-Erkrankungen [Selik et al. Abstr. 871].

Trotz der gewachsenen klinischen Erfahrung und der verbesserten Therapieregime gibt es aber weiterhin Therapieversagen. In der EuroSIDA-Kohorte wurde nach Faktoren gesucht, die ein Versagen aller 3 Medikamentenklassen begünstigen. Ein wichtiger Faktor ist die Vorbehandlung mit weniger wirksamen Mono- und Zweifachtherapien. Von den 3Klassenversagern kommen 400 aus der Gruppe der 2.430 vortherapierten Patienten $(16,5 \%)$ und 69 aus der Gruppe der 1.108 vor HAART-Beginn therapienaiven Patienten (6,2\%). 6 Jahre nach Beginn einer HAART lag bei $24 \%$ der vorbehandelten, aber nur bei $12 \%$ der therapienaiven Patienten ein 3-Klassenversagen vor. Die Versagerrate stieg mit zunehmender Behandlungsdauer. Bei therapienaiven $\mathrm{Pa}$ tienten betrug sie 1,2/100 Personenjahre in den ersten 2 Jahren und stieg dann auf 4,7/100 Personenjahre nach 5 und mehr Jahren Behandlung. Bei NRTI-vorbehandelten Patienten wurde das Risiko eines Therapieversagens reduziert, wenn bei Beginn einer HAART auch auf neue NRTIs umgestellt worden war. Auch die Dau- 
er der NRTI-Vorbehandlung beeinflusste die Geschwindigkeit des Therapieversagens [Mocroft et al. Abstr. 554].

\section{Klinische Komplikationen unter HAART}

Während opportunistische Infektionen und das Kaposi-Sarkom bei erfolgreicher antiretroviraler Therapie deutlich seltener werden, können bestimmte Krebserkrankungen an Häufigkeit zunehmen. In San Diego wurde ein Abgleich zwischen AIDSund Krebsregister vorgenommen, um die Entwicklung der Analkarzinomhäufigkeit bei AIDS-Patienten zu verfolgen. Analysiert wurden die Daten aus dem Zeitraum von 1988-2000. In dieser Zeitspanne wurden 39 Analkarzinome bei AIDS-Patienten registriert, nahezu ausschließlich bei Männern mit gleichgeschlechtlichen Sexualkontakten [38]. Im Zeitraum zwischen 1988 und 1996 wurden nur 8 Fälle registriert, danach stieg die Zahl von Jahr zu Jahr kontinuierlich an: 3 Fälle im Jahr 1996, 4 im Jahr 1997, 5 im Jahr 1998, 8 im Jahr 1999 und 11 Fälle im Jahr 2000. Bezogen auf 1.000 AIDS-Fälle verzehnfachte sich die Rate von 2,8 Analkarzinomen im Jahr 1992 auf 24,7 Fälle im Jahr 2000. Es ist unwahrscheinlich, dass die erhöhte Erkrankungsrate durch ein intensiviertes Screening bedingt ist, da der Anteil der Karzinome in situ in der Vor-HAART-Ära 38\% (3/8), in der Nach-HAART-Ära dagegen nur 26\% (8/31) betrug. Die plausibelste Erklärung für die Zunahme der Analkarzinome ist die längere Überlebensdauer von AIDS-Patienten nach Einführung der HAART. Während früher Patienten bereits vor einer Manifestation des Analkarzinoms an opportunistischen Infektionen verstarben, erleben sie diesen Zeitpunkt jetzt noch. Die Pathogenese des Analkarzinoms scheint im Gegensatz zu opportunistischen Infektionen durch die antiretrovirale Kombinationstherapie nicht oder nicht im selben Maße beeinflusst zu werden [Diamond et al. Abstr. 778]. Eine ähnliche Entwicklung scheint sich bei einer Reihe anderer, nicht AIDS-definierender Krebserkrankungen anzubahnen. In der HIV Outpatient Study (HOPS) wurde die Inzidenz folgender Krebsdiagnosen bei HIV-Patienten im Vergleich zur Allgemeinbevölkerung bestimmt: Lungenkarzinom, Mor- bus Hodgkin, Analkarzinom, malignes Melanom und Krebs der oberen Luft- und Speisewege. Nach Adjustierung für Alter, Geschlecht, ethnische Zugehörigkeit und Rauchen war die Inzidenz von Lungenkarzinomen (relatives Risiko/RR=2,13), malignen Melanomen $(\mathrm{RR}=2,99)$, Morbus Hodgkin $(\mathrm{RR}=4,58)$ und Analkarzinomen $(\mathrm{RR}=10,13)$ bei HIV-Patienten deutlich höher als in der Allgemeinbevölkerung [Patel et al. Abstr. 81].

\section{Langzeitnebenwirkungen der HAART}

Um Langzeitnebenwirkungen einer HAART vor allem in Bezug auf Herz-Kreislauf-Erkrankungen zu analysieren, werden entsprechende Verlaufsdaten mehrerer großer Kohortenstudien im Rahmen der D:A:D-Studie analysiert. Eine bereits veröffentlichte Analyse fand eine pro Jahr unter antiretroviraler Kombinationstherapie um 26\% erhöhte Inzidenz von Herzinfarkten. Eine weitere, jetzt in San Francisco vorgestellte Analyse beschäftigt sich mit der Inzidenz eines breiteren Spektrums kardiovaskulärer Komplikationen (Herzinfarkt, Herz-Kreislauf-Versagen, invasiver kardiovaskulärer Eingriff, Schlaganfall). Auch in dieser Analyse zeigt sich ein mit der Dauer einer HAART-Behandlung ansteigendes Risiko [Law et al. Abstr. 737].

Eine weitere Analyse der D:A:D-Studie galt dem Blutdruck. Bluthochdruck ist ein bekannter Risikofaktor für kardiovaskuläre Erkrankungen. Es wurde postuliert, dass Patienten unter HAART ein diesbezüglich erhöhtes Risiko aufweisen. Diese Behauptung konnte durch die Analyse nicht bestätigt werden. Die Entwicklung eines Bluthochdrucks war durch klassische Risikofaktoren wie Alter, Geschlecht und Body-Mass-Index zu erklären. Es fand sich keine Assoziation zwischen Blutdruck und kumulativer Dauer der Exposition gegenüber antiretroviralen Medikamenten [Thiébaut et al. Abstr. 75]. Zu den Stoffwechselveränderungen, die vermehrt unter einer HAART beobachtet werden, zählt auch eine erhöhte Insulinresistenz, die zu Hyperglykämien und zum Diabetes mellitus führen kann. Im Rahmen der Multicenter AIDS Cohort Study (MACS) wurde die Inzidenz von Hyperglykämien und Diabetes mellitus bei HIV-infizierten und HIV-negativen homosexuellen Männern bestimmt. Von den 544 HIV-infizierten Teilnehmern werden inzwischen 423 mit einer HAART behandelt. Analysiert wurden Daten, die zwischen April 1999 und September 2002 erhoben worden waren. Bereits bei der ersten Untersuchung waren im Vergleich zur Kontrollgruppe (5\%) mit 14\% fast 3-mal so viele HIV-Infizierte an einem Diabetes mellitus erkrankt. Während der 3,5-jährigen Studiendauer entwickelten 79 Teilnehmer erstmals Hyperglykämien, davon 38 einen manifesten Diabetes mellitus. HAART-behandelte Patienten zeigten eine 1,8-mal höhere Wahrscheinlichkeit für die Ausprägung erhöhter Blutzuckerwerte und ein 3,5fach höheres Risiko für die Entwicklung eines Diabetes mellitus. Ein erhöhtes Risiko besaßen vor allem Patienten, die mit Proteaseinhibitoren, Stavudin oder Efavirenz behandelt worden waren [Brown et al. Abstr. 73]. In mehreren Studien wurde untersucht, ob sich bestimmte Therapieregime bezüglich ihrer Auswirkungen auf den Glukose- und Fettstoffwechsel unterscheiden. Die A5005-Studie verglich diesbezüglich die Auswirkungen einer Nelfinavir mit denen einer Efavirenz enthaltenden Therapie und die Effekte von Zidovudin+Lamivudin mit denen von Didanosin+Stavudin. Unter Nelfinavir und Efavirenz wurde ein vergleichbarer Anstieg in den Blutfetten (nüchtern) beobachtet. Die Veränderungen der Blutfette fielen unter Didanosin+Stavudin etwas ungünstiger aus als unter Zidovudin+Lamivudin. Eine Erhöhung der Insulinresistenz wurde unter allen Therapieregimen beobachtet (ohne signifikante Unterschiede zwischen den Regimen) [Dubé et al. Abstr. 74].

Der Wechsel von einer PI-enthaltenden auf eine NNRTI-enthaltende Kombination oder eine Tripel-NRTI-Therapie war die erste Strategie, die zur Vermeidung der Stoffwechselveränderungen erprobt wurde. Ziel der 3-armigen spanischen NEFAStudie, in der bei virologisch erfolgreich behandelten Patienten der Proteaseinhibitor durch Nevirapin, Efavirenz oder Abacavir ersetzt worden war, war zwar vorrangig die Therapievereinfachung, untersucht wurden aber auch die Auswirkungen auf den Fettstoffwechsel. Nach dem Wechsel verbesserten sich in allen 3 Therapiearmen die Insulinwerte und Insulin- 


\section{Tagungsbericht}

resistenz sowie die Low-Density-Lipoprotein- und High-Density-Lipoproteinwerte, die Triglyzeridspiegel blieben unverändert (- Tabelle 2) [Fisac et al. Abstr. 78].

Eine wesentliche Verbesserung von Symptomen der Körperfettumverteilung (Lipoatrophie oder Lipohypertrophie) wurde nach dem Therapiewechsel nicht beobachtet. Im Hinblick auf die virologische Wirksamkeit wurden im Abacavir-Arm die meisten Therapieversager beobachtet, Nevirapin und Efavirenz unterschieden sich diesbezüglich nicht. Die Mehrzahl der nach Umstellung virologisch versagenden Patienten waren vor einer HAART mit suboptimalen Nukleosidanalogazweifach- oder Monotherapien behandelt worden. Die Verträglichkeit der beiden NNRTI-Arme war dagegen etwas schlechter als die des Abacavir-Armes: Es traten die zu erwartenden kutanen, lebertoxischen und neuropsychiatrischen Nebenwirkungen auf, die in den beiden NNRTI-Armen bei jeweils $17 \%$ der Teilnehmer zu einer erneuten Umstellung der Therapie führten [Martinez E et al., N Eng J Med 2003].

In der internationalen MaxCmin2-Studie wurden Unterschiede im Lipidprofil nach Behandlung mit Lopinavir bzw. Saquinavir, jeweils mit Ritonavir geboostet, untersucht. Im Lopinavirarm stiegen die Triglyzeridspiegel um fast 30\%, während sie im Saquinavirarm um knapp 6\% zurückgingen. Die Gesamtcholesterinwerte stiegen in beiden Armen an, blieben jedoch auch nach 48 Wochen Behandlung noch im normalen Bereich [Walmsley et al. Abstr. 720]. Verglichen wurden auch Lopinavir und der neue Proteaseinhibitor Atazanavir im Hinblick auf ihren jeweiligen Einfluss auf den Glukosestoffwechsel. In einer randomisierten doppelblinden und placebokontrollierten Cross-over-Studie an gesunden Probanden wurde der Effekt der beiden PIs auf den Glukosetransport ins Gewebe untersucht. Atazanavir reduzierte dabei im Gegensatz zu Lopinavir die Insulinsensitivität nicht [Noor et al. Abstr. 702].

In einer Substudie der Atlantic-Studie (IDV vs. NVP vs. 3TC, jeweils kombiniert mit d4T+ddI) wurde untersucht, ob sich die 3 Behandlungsarme in Bezug auf Lipidprofile und Fettgewebsverteilungsstörungen voneinander unterscheiden. Eine Lipoatrophie wurde in allen 3 Behandlungsarmen mit gleicher Häufigkeit fest-

Tabelle 2

Veränderungen der Blutfettwerte gegenüber Studienbeginn 2 Jahre nach Austausch des Protease-Inhibitors (PI) in der NEFA-Studie

\begin{tabular}{lccc} 
& NVP-Arm & EFV-Arm & ABC-Arm \\
\hline Gesamtcholesterin & $+2 \%$ & $-6 \%$ & $-13 \%$ \\
\hline LDL & $-17 \%$ & $-10 \%$ & $-15 \%$ \\
\hline HDL & $+50 \%$ & $+12 \%$ & \\
\hline Gesamtcholesterin/HDL & $+16 \%$ & $+15 \%$ & $+5 \%$
\end{tabular}

NVP Nevirapin, EFV Efavirenz, ABC Abacavir.

gestellt (39\% in Woche 192), viszerale Fetthypertrophie war im Indinavir-Arm etwas häufiger. Die HDL-Werte entwickelten sich im Nevirapin-Arm am vorteilhaftesten [Murphy et al. Abstr. 718]. Auch das Glukose-Insulin-Profil von Nevirapin war in einer Vergleichsuntersuchung günstiger als das von Protease-Inhibitoren und von Efavirenz [Shahmanesh et al. Abstr. 704]. Beim Vergleich der Stoffwechseleffekte von Emtricitabin und Stavudin, jeweils kombiniert mit Didanosin und Efavirenz, schneidet Emtricitabin sowohl was den Glukose- und Lipidstoffwechsel, als auch die Körperfettverteilung angeht, besser ab als Stavudin [Powderly et al. Abstr. 717]. Schließlich wurden in den Enfurvitide-Zulassungsstudien TORO 1 und 2 auch noch Ergebnisse einer Untersuchung auf Fettverteilungsstörungen, Lipid- und Glukosestoffwechselveränderungen vorgestellt. Dieser neue Fusionsinhibitor scheint glücklicherweise keine Auswirkungen auf die untersuchten Parameter zu haben [Cooper et al. Abstr. 715].

Zur Erhellung der Ursachen der Fettgewebsverteilungsstörungen wurden HIVnegative Versuchspersonen in einer australischen Studie mit Stavudin+Lamivudin oder Zidovudin+Lamivudin über einen Zeitraum von 6 Wochen behandelt. Bereits nach 2 Wochen nahm die Expression mitochondrialer Gene und des PPAR Gens in Fettgewebe deutlich ab. Dieser Effekt war unter Stavudin stärker ausgeprägt als unter Zidovudin [Mallon et al. Abstr. 76]. Der Gehalt an mitochondrialer DNA in Fettgewebe und mononukleären Blutzellen wurde in einer weiteren Studie an Patienten mit und ohne Lipoatrophie bestimmt. Während niedrigere mitochondriale DNA-Werte im Fettgewebe mit Lipoatrophie assoziiert war, fand sich keine diesbezügliche Korrelation mit dem Gehalt mitochondrialer DNA in Blutzellen, sodass sich diese nicht als Surrogatmarker für die mitochondriale Toxizität auf Fettzellen eignen [Gerschenson et al. Abstr. 710]. Eine fehlende Korrelation des Gehalts an mitochondrialer DNA in Blutzellen mit klinischen Symptomen oder Laborparametern für eine mitochondriale Toxizität wird auch aus einer niederländischen Studie berichtet [Casula et al. Abstr. 709].

\section{Virale Resistenzentwicklung und Resistenzübertragung}

\section{Resistenzentwicklung}

In den letzten Monaten wurde über schlechte Behandlungsergebnisse mit verschiedenen Tripel-Nukleosid/Nukleotidanaloga-Kombinationen berichtet. Allen diesen Kombinationen war gemeinsam, dass sie kein Thymidinanalogon (Zidovudin oder Stavudin) enthielten und ihre Anwendung in hohen Prozentsätzen zu viralen M184V- und/oder K65R-Mutationen führte (• Abb. 2) [Jemsek et al. Abstr. 51; Landman et al. Abstr. 52]. Die plausibelste Erklärung für das frühe virologische Versagen dieser Dreifachkombinationen ist das Vorliegen einer niedrigen genetischen Barriere gegen die Resistenzentwicklung. Bislang gibt es keine Hinweise auf mögliche andere Ursachen, wie $\mathrm{z}$. B. eine gegenseitige Beeinflussung der Plasma- oder der intrazellulären Wirkspiegel.

In einer Studie, die die Wirksamkeit einer einmal täglichen Gabe von Trizivir $^{2}+$ Tenofovir untersucht, wurde nach

\footnotetext{
${ }^{2}$ Trizivir ist eine fixe Kombination von 3 Nukleosidanaloga: Abacavir, Lamivudin und Zidovudin (ZDV)
} 


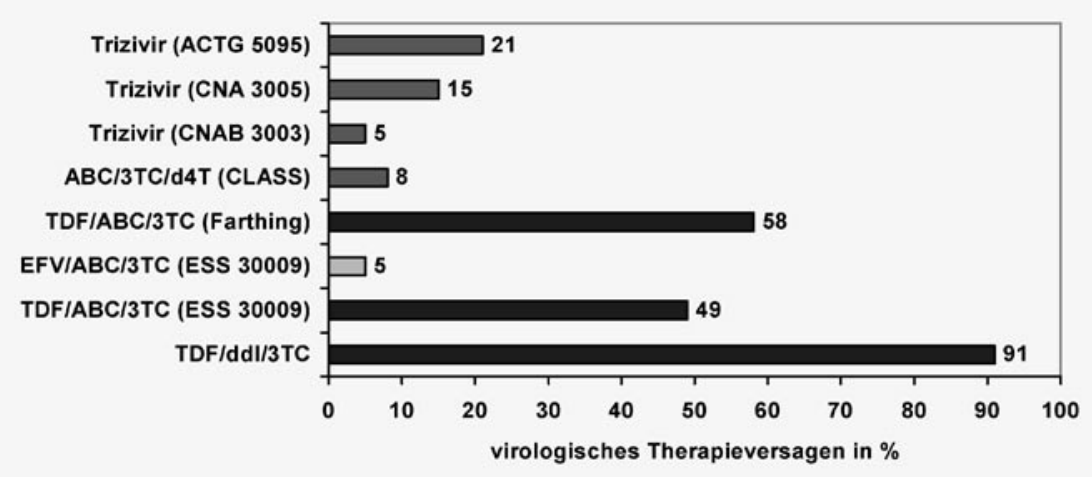

Abb. $2 \triangle$ Raten virologischen Therapieversagens in verschiedenen Studien mit Tripel-Nukleosid/ Nukleotidanaloga-Kombinationen. ABC Abacavir, EFV Efavirenz, ddI Didanosin, 3TC Lamivudin, d4t Stavudin, TDF Tenfovir

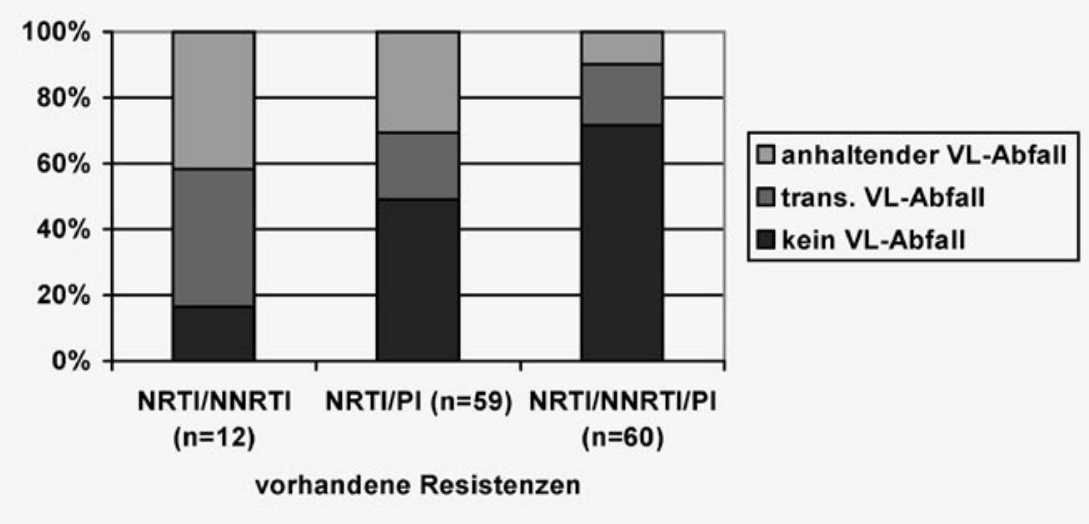

Abb. $3 \triangle$ Ansprechraten auf Therapieumstellung in Abhängigkeit vom vorhandenen viralen Resistenzmuster. VL Viruslast, NRTI nukleosidische Reverse Transkriptase-Inhibitoren, NNRTI nicht nukleosidische Reverse Transkriptase-Inhibitoren, PI Protease-Inhibitor

dem vorzeitigen Abbruch einer weiteren Studie mit der Kombination Tenofovir+Abacavir+Lamivudin eine Zwischenanalyse vorgenommen. Die 24-WochenWirksamkeitsdaten bewegten sich im erwarteten Bereich: 78\% der Teilnehmer erreichten eine Viruslast von $<400$ Kopien/ $\mathrm{ml}, 67 \%$ eine Viruslast von $<50$ Kopien. Ein frühes virologisches Versagen $(<2 \mathrm{log}$ Viruslastabfall in den ersten 8 Wochen) wurde bei $11 \%$ der Teilnehmer registriert. Die Mehrzahl dieser Patienten besaß eine Ausgangsviruslast von $>100.000$ Kopien. Eine virale K65R-Mutation wurde bei Therapieversagern nur in einem Fall festgestellt, in den anderen Fällen wurden Wildtypvirus oder sog. Thymidinanalogamutationen (TAMs) mit oder ohne M184V-Mutation beobachtet [Elion et al. Abstr. 53]. Obwohl die Zidovudin-Dosierung bei einmal täglicher Gabe von Trizivir+Tenofovir wahrscheinlich zu gering ist, scheint es in dieser Kombination die Entwicklung der viralen K65R-Mutation zu verhindern. Darauf deutet auch eine Analyse von viralen Resistenzmustern in der Virco-Datenbank und eine Suszeptibilitätstestung von Virusisolaten mit definierten Resistenzmustern hin. Die Häufigkeit der K65R-Mutation stieg von $0,8 \%$ der Virusisolate im Jahr 1998 auf 3,8\% im Jahr 2003. Virusisolate mit der K65R-Mutation zeigten ein vermindertes Ansprechen auf alle Nukleosidanaloga außer Zidovudin. Die ZDV-typischen Mutationen 41L, 67N, 210W und $215 \mathrm{Y} / \mathrm{F}$ traten nur sehr selten gemeinsam mit einer 65R-Mutation auf. Der vermutete Antagonismus von 65R und TAMs konnte dadurch bestätigt werden, dass das Einbringen einer 65R-Mutation in ein Virus mit 3 oder 4 TAMs zu einem verbesserten Ansprechen auf ZDV sowie auf ddC, ddI, d4T, Abacavir und Tenofovir führte [Parikh et al. Abstr. 54].
Anhand größerer Therapiekohorten wurde das Risiko der viralen Resistenzentwicklung unter bestimmten Therapiekombinationen analysiert. Bei der genotypischen Resistenztestung von 2.324 Patienten aus Nordkalifornien wurden 74 Patienten $(3,2 \%)$ mit hochgradiger NRTI/PI-2Klassenresistenz, 21 Patienten (0,9\%) mit hochgradiger NRTI/NNRTI-Resistenz und 82 Patienten (3,5\%) mit einer 3-Klassenresistenz identifiziert. Bei Therapieumstellung zeigten sich in Abhängigkeit vom vorhandenen Resistenzmuster unterschiedliche Ansprechraten (• Abb. 3).

Hochgradige 2- oder 3-Klassenresistenzen wurden fast ausschließlich bei Personen beobachtet, die vor der HAART-Einführung mit Mono- oder Zweifachtherapien behandelt worden waren. Eine Umstellung ist erfolgreicher, wenn noch eine neue Medikamentenklasse zur Verfügung steht, aber die Erfolge sind oft nur vorübergehender Natur. Ob durch die Umstellung auf ein Regime mit 2 neuen Medikamentenklassen (inklusive Fusionsinhibitoren) der längerfristige Behandlungserfolg verbessert werden kann, bedarf weiterer Studien [Fessel et al. Abstr. 690].

In einer kanadischen Studie wurden die viralen Resistenzentwicklungsmuster bei knapp 1.100 zuvor therapienaiven Patienten analysiert. In PI/NRTI-haltigen Kombinationen war die 3TC-Resistenz (M184 V) die sich am schnellsten und häufigsten entwickelnde Resistenz, in NNRTIhaltigen Kombinationen waren es NNRTIResistenzen [Harrigan et al. Abstr. 689]. Vergleichbare virale Resistenzmuster werden in der CNA30024-Studie beschrieben, die Wirksamkeit und Sicherheit der beiden Kombinationen $\mathrm{ZDV}+3 \mathrm{TC}+\mathrm{EFV}$ und $\mathrm{ABC}+3 \mathrm{TC}+\mathrm{EFV}$ bei zuvor therapienaiven Teilnehmern vergleicht. Auch in dieser Studie liegen bei Therapieversagern häufiger NNRTI-Mutationen als M184VMutationen vor (• Tabelle 3 ) [Irlbeck et al. Abstr. 661].

Eine Analyse der 6-armigen ACTG384-Studie, die 4 Dreifachkombinationen mit 2 Vierfachkombinationen verglich, zeigte, dass der virologisch erfolgreichste Arm (ZDV+3TC+EFV) auch die niedrigste Rate an Resistenzentwicklung aufwies (10\%). Unter einer d4T/ddI-Kombination wurde die Resistenzmutation 65R und $74 \mathrm{~V}$ selektiert, aber nicht die $184 \mathrm{~V}$-Mutati- 
on. Eine nachweisbare genotypische Resistenz war allerdings (wie auch in der oben beschriebenen CNA-30024-Studie) nicht die einzige Ursache für ein virologisches Versagen, da in vielen Fällen keine Resistenzmutationen entdeckt werden konnten. Welche Ursache das Versagen hier hatte, bedarf weiterer Untersuchungen [Johnson et al. Abstr. 662].

Die Frage, ob nach dokumentierter viraler Resistenzentwicklung die entsprechenden Medikamente noch eine Restaktivität entfalten oder abgesetzt werden können, wurde bei einer Reihe von Patienten durch Absetzen einzelner Substanzen aus einem Therapieregime geprüft. Die Viruslast wurde nach Absetzen von Stavudin und Didanosin über einen Zeitraum von $14 \mathrm{Ta}$ gen, für Efavirenz über einen Zeitraum von 3 Wochen bestimmt. Nach Absetzen von Stavudin kam es bei 3/3 Patienten zu einem deutlichen Anstieg der Viruslast, nach Absetzen von Efavirenz (vorhandene Mutationen: K103N und Y188L) und Didanosin (Mutationen: M41L, L210W und T215Y) änderte sich die Viruslast hingegen nicht. Das deutet darauf hin, dass Stavudin auch bei Vorliegen von viralen Resistenzmutationen noch eine Restaktivität entfalten kann, Efavirenz und Didanosin jedoch nicht. Allerdings stellt sich die Frage, ob angesichts der langen Halbwertzeit von Efavirenz ein Zeitraum von 3 Wochen ausreicht, um einen Effekt nachzuweisen [Maldarelli et al. Abstr. 623].

Bei Patienten, bei denen aufgrund ihrer Behandlungsvorgeschichte eine Unterdrückung der Virusreplikation unter die Nachweisgrenze nicht mehr möglich ist, führt eine HAART-Behandlung bei guter Therapietreue zu einer weiteren Akkumulation von viralen Resistenzmutationen. Auf der anderen Seite bedingt ein Behandlungsabbruch die Rückkehr des Wildtypvirus mit seiner gegenüber resistenten $\mathrm{Vi}$ rusvarianten oft erhöhten Replikationsfähigkeit und Pathogenität. Eine französische Arbeitsgruppe untersuchte daher, ob das Virus durch eine kalkulierte Reduktion des medikamentösen Selektionsdrucks auf dem erreichten Evolutionsstand „eingefroren“ werden kann. Ihr Ziel war die Beibehaltung eines durch Resistenzmutationen in seiner Fitness geschwächten Virus und die Vermeidung weiterer, diese wieder verbessernde Mutationen. Um die-

Tabelle 3

Virales Resistenzentwicklungsmuster unter NRTI-NNRTI-Kombinationen

\begin{tabular}{|lll}
\hline & ZDV+3TC+EFV & ABC+3TC+EFV \\
\hline $\mathrm{VL}>400$ in Woche 48 & $7 / 325$ & $11 / 324$ \\
\hline $\mathrm{VL}>50$ in Woche 48 & $13 / 325$ & $20 / 324$ \\
\hline Genotypische Resistenzen $(\mathrm{VL}>500)$ & Wildtyp 3x & Wildtyp 7x \\
& $103 \mathrm{~N}+184 \mathrm{~V}$ & $103 \mathrm{~N}(2 \mathrm{x})$ \\
& $103 \mathrm{~N}+184 \mathrm{~V}+190 \mathrm{~A}$ & $184 \mathrm{~V}+190 \mathrm{~S}$ \\
& $103 \mathrm{~N}+184 \mathrm{~V}+225 \mathrm{H}$ & $103 \mathrm{~N}+184 \mathrm{~V}+225 \mathrm{H}$ \\
& $103 \mathrm{~N}+108 \mathrm{I}+184 \mathrm{~V}$ & \\
\hline
\end{tabular}

VL Viruslast, NRTI nukleosidische Reverse Transkriptase-Inhibitoren, NNRTI nicht nukleosidische Reverse Transkriptase-Inhibitoren, ZDV Zidovudin, 3TC Lamivudin, EFV Efavirenz, ABC Abacavir.

Tabelle 4

Ansprechraten auf eine NNRTI-haltige Kombinationstherapie bei Frauen mit und ohne vorherige Nevirapin-Kurzprophylaxe und mit oder ohne nachgewiesene virale NNRTI-Resistenzen nach der Prophylaxe

\begin{tabular}{|llll}
\hline & $\begin{array}{l}\text { NVP-induzierte } \\
\text { Mutation }\end{array}$ & $\begin{array}{l}\text { NVP-exponiert } \\
\text { ohne Mutation }\end{array}$ & $\begin{array}{l}\text { Nicht NVP- } \\
\text { exponiert }\end{array}$ \\
\hline $\mathrm{VL}<400$ nach 3 Monaten Therapie & $80 \%$ & $87 \%$ & $88 \%$ \\
\hline $\mathrm{VL}<50$ nach 3 Monaten & $45 \%$ & $46 \%$ & $54 \%$ \\
\hline $\mathrm{VL}<400$ nach 6 Monaten Therapie & $68 \%$ & $80 \%$ & $85 \%$ \\
\hline $\mathrm{VL}<50$ nach 6 Monaten & $38 \%$ & $50 \%$ & $74 \%$ \\
\hline $\begin{array}{l}\text { Therapiebeginn früher als 6 Monate } \\
\text { nach Entbindung, } \mathrm{VL}<400\end{array}$ & $\mathbf{5 8 \%}$ & $69 \%$ & \\
\hline $\begin{array}{l}\text { Therapiebeginn später als 6 Monate } \\
\text { nach Entbindung, } \mathrm{VL}<400\end{array}$ & $\mathbf{7 7 \%}$ & $91 \%$ & \\
\hline
\end{tabular}

VL Viruslast, NNRTI nicht nukleosidische Reverse Transkriptase-Inhibitoren, NVP Nevirapin.

ses Ziel zu erreichen, wurden die Studienteilnehmer $(n=26)$ mit einer Kombination aus niedrig dosiertem Indinavir+Ritonavir (200/100 mg 2-mal/Tag) und Lamivudin (150 mg 2-mal/Tag) behandelt. Im Verlauf der 24-wöchigen Studie erlebten 3 Teilnehmer ein virologisches Versagen (>0,7 log-Anstieg über Ausgangswert), 6 Teilnehmer ein immunologisches Versagen $(>25 \%$ Abfall der CD4-Zellzahl) und ein Teilnehmer beides. Bei den 21 Teilnehmern, deren Daten ausgewertet werden konnten, lag die Viruslast um 0,22 log höher als zu Beginn der Studie. Die CD4-Zellzahl verringerte sich im Mittel um 49 Zellen. Verglichen mit dem Absinken der Zellzahl unter dem versagenden Therapieregime war der Zellverlust aber nur unwesentlich höher (10 Zellen/Monat vs. 7 Zellen/Monat). Die genotypischen Veränderungen im Protease-Gen waren gering (eine zusätzliche M46L- und eine I54V-Muta- tion, einmal Verlust der G73S-Mutation), im RT-Gen entwickelten 2 Teilnehmer eine zuvor nicht vorhandene M184V-Mutation. Da es sich bei der Untersuchung aber noch um eine unkontrollierte Pilotstudie handelt, sind diese Ergebnisse nur schwer zu interpretieren. [Launay et al. Abstr. 649].

Eine andere französische Gruppe untersuchte den Beitrag von Minderheitspopulationen des Virus, die bei üblichen Resistenztests unterhalb der Nachweisgrenze bleiben, auf die weitere Resistenzentwicklung bei Therapieumstellung. Durchgeführt wurden die Untersuchungen an $8 \mathrm{~Pa}$ tienten, die nach mehrfachem Versagen von PI-haltigen Therapieregimen auf eine Salvage-Therapie mit Lopinavir+Amprenavir umgestellt worden waren, auch hier virologisch versagten und zusätzliche $\mathrm{Re}$ sistenzmutationen entwickelten. Von den insgesamt 19 neuen Mutationen, die sich 
bei diesen Patienten bis zur 26. Woche nach Therapieumstellung entwickelt hatten, waren 4 bereits nach 2 Wochen, weitere 5 nach 6 Wochen nachweisbar. Um Minderheitspopulationen nachzuweisen, wurden die viralen Proteasesequenzen vor Umstellung der Therapie amplifiziert und kloniert. Die Mutationen, die nach 2 Wochen nachweisbar wurden, lagen bereits vor Umstellung als Minderheitspopulationen vor. Bei den später entdeckten Mutationen waren diese nur in einem Fall schon im Ausgangsviruspool nachweisbar [Charpentier et al. Abstr. 57].

Japanische Wissenschaftler analysierten die Rolle, die Gag-Mutationen für die virale Fitness und die Proteaseinhibitorresistenz spielen. Virus, das 6 Proteasemutationen trug, aber keine Gag-Mutationen, war in der Zellkultur nicht replikationsfähig. Im Gegensatz dazu war Virus, das ausschließlich Gag-Mutationen aufwies, genauso replikationskompetent wie das Wildtypvirus. Werden Viren mit Gag-Mutationen mit einem Proteaseinhibitor behandelt, können sich Proteasemutationen z. T. schneller entwickeln als beim Wildtypvirus [Koh et al. Abstr. 634]. Aus Brasilien wurde über Subtypabhängige Unterschiede bei der viralen Resistenzentwicklung berichtet: Zellkulturstudien zeigen, dass die L89M-Mutation in Abhängigkeit vom viralen Subtyp (Subtyp F oder Subtyp B) unterschiedliche Auswirkungen auf die Resistenz und Fitness eines Virus hat [Calazans et al. Abstr. 692].

\section{Persistenz und Übertragung von Resistenzen}

Die potenziellen Konsequenzen der Resistenzinduktion gegen NNRTIs durch die Nevirapin-Kurzprophylaxe zur Reduktion der Mutter-Kind-Übertragungsrate von HIV sind sehr weit reichend: NNRTI-haltige Kombinationspräparate sind nach den Behandlungsempfehlungen der WHO in Entwicklungsländern die bevorzugte Initialtherapie. Falls diese Therapieregime bei einem erheblichen Teil der $\mathrm{zu}$ behandelnden Frauen nicht mehr ihre volle Wirksamkeit entfalten können, würde das die Erfolgsaussichten einer umfassenden HAART-Einführung in diesen Ländern spürbar beeinträchtigen.
In einer südafrikanischen Studie wurde die hohe Rate von Resistenzinduktionen bei Schwangeren durch die Kurzprophylaxe erneut bestätigt. Untersuchungen 4-10 Wochen nach der Entbindung zeigten, dass 44\% der Mütter mindestens eine virale NNRTI-Resistenzmutation aufwiesen, in den Folgewochen sank der Anteil der Frauen, bei denen diese Mutation nachweisbar blieb, auf 24\% [Martinson et al. Abstr. 38].

In Thailand wurde bei Frauen, die im Rahmen einer Studie zusätzlich zur Zidovudin-Prophylaxe eine einmalige Nevirapin-Dosis erhalten hatten, das Ansprechen auf eine nach der Entbindung begonnene NNRTI-haltige Kombinationstherapie analysiert. Nach der Entbindung war bei $18 \%$ der Frauen eine NNRTI-Resistenzmutation nachweisbar. Der virologische Erfolg der Kombinationstherapie war in der Gruppe, die nach der Einmaldosis eine Resistenzmutation entwickelte, am geringsten (• Tabelle 4), obwohl ein erheblicher Anteil dieser Frauen auch in Gegenwart der Mutationen noch ein gutes virologisches Ansprechen zeigte. Zudem scheint ein längerer zeitlicher Abstand zwischen der Kurzprophylaxe und dem Therapiebeginn aufgrund einer mütterlichen Indikation den Behandlungserfolg zu verbessern. Längere und umfangreichere Verlaufsbeobachtungen sind erforderlich, um die Konsequenzen der Nevirapin-Kurzprophylaxe auf die mütterliche Therapieoptionen besser einschätzen zu können [Jourdain et al. Abstr. 41LB].

Ein Problem der Resistenztestung liegt darin, dass Resistenzen tragende virale Teilpopulationen nur dann nachgewiesen werden, wenn sie einen ausreichend hohen Anteil der Gesamtpopulation ausmachen. Von den Standardtests werden üblicherweise Teilpopulationen, die weniger als 20-25\% der Gesamtpopulation ausmachen, nicht mehr sicher erkannt. Natürlich stellt sich die Frage, ob kleinere resistente Teilpopulationen nicht auch zu einem virologischen Therapieversagen beitragen können. Im Rahmen der ACTG-398-Studie, in der NNRTI-erfahrene und NNRTI-naive Patienten auf eine Kombination aus Efavirenz, Abacavir, Adefovir und Amprenavir士einem weiteren PI umgestellt worden waren, wurde in einer Ausgangsblutprobe nach solchen Populationen gesucht. Bei
48/212 NNRTI-erfahrenen Patienten wurden mit einem verfeinerten Verfahren virale NNRTI-Mutationen entdeckt, die in einem genotypischen Standardresistenztest nicht aufgefallen waren. Das virologische Ansprechen bei diesen Patienten mit unterschwelliger viraler Resistenz war genauso schlecht wie bei Patienten mit im Standardtest nachweisbarer Resistenz. Eine Resistenz kann also bereits vorhanden, aber mit Standardmethoden noch nicht nachweisbar sein, wenn eine Vorbehandlung erfolgt ist [Mellors et al. Abstr. 39]. In einer weiteren Studie wurde untersucht, wie lange NNRTI-Resistenzmutationen nach virologischem Versagen eines NNRTI-haltigen Therapieregimes und Absetzen des NNRTI nachweisbar sind. Die bei Therapieversagen dominierende K103N-Mutation blieb bei den Patienten über Zeiträume von 2-5 Jahren detektierbar. Da sich die Fitness der resistenten viralen Variante offenbar kaum vom Wildtypvirus unterscheidet, besteht für diese keine Notwendigkeit, zurück zu mutieren [Palmer et al. Abstr. 37].

Während Virus mit NRTI- und PI-Resistenzmutationen, die sich unter Behandlung herausbilden und die virale Fitness beeinträchtigen, nach Absetzen der entsprechenden Substanzen wieder durch „archiviertes“ Wildtypvirus ersetzt wird, kann bei Infektionen mit einem primär resistenten Virus eine andere Situation vorliegen: In diesem Fall ist kein Wildtypvirus vorhanden, das resistente Virus müsste also zurück mutieren um seine Resistenzmutationen zu verlieren. Bei Vorliegen einer Punktmutation ist dies relativ problemlos möglich, bei multiplen Resistenzmutationen kann dies aber ein unüberwindliches Problem darstellen, da das Virus nicht in einem Schritt, sondern über verschiedene Zwischenstufen mutieren müsste. Zudem müsste es in vielen Fällen ein „Fitnesstal“ überwinden, was ohne Selektionsdruck von außen nicht gelingt. Dies bedeutet, dass übertragene komplexe Resistenzen noch Jahre nach dem Infektionsereignis nachweisbar bleiben. Ein Resistenztest vor Therapiebeginn kann daher auch bei chronisch infizierten Patienten, deren Infektionszeitpunkt bereits länger zurückliegt, sinnvoll sein [Cane et al. Abstr. 684; Little et al. Abstr. 36LB].

In einer multizentrischen europäischen Untersuchung wurde das Therapieanspre- 
chen bei Serokonvertern untersucht, die sich mit primär resistenten Virusvarianten infiziert hatten. Das initiale Therapieregime war bei diesen Patienten in der Regel an den Resistenzstatus adaptiert worden. Die Ansprechrate auf eine resistenzadaptierte HAART unterschied sich nicht von der Ansprechrate auf eine Standard-HAART bei Patienten mit Wildtypvirus [Pillay et al. Abstr. 685]. Inwiefern die längerfristigen Therapieoptionen durch die übertragene Resistenz beeinträchtigt werden, bleibt abzuwarten.

Aus Amsterdam und North-Carolina wurde übereinstimmend über einen Rückgang übertragener Primärresistenzen berichtet: In North-Carolina wurden in den Jahren von 1998-2000 Resistenzen bei 4 von 12 frisch infizierten Patienten festgestellt, im Zeitraum von 2000-2003 fand sich unter 18 Primärinfektionen hingegen kein Fall [Hicks et al. Abstr. 682]. In Amsterdam lag zwischen 1994 und 1998 der Anteil von Primärresistenzen in einer Größenordnung von 13\%, bei den danach diagnostizierten Serokonversionen nur noch bei 6\% [Bezemer et al. Abstr. 679]. Die Ursache für den Rückgang der Primärresistenzen ist der Trend $\mathrm{zu}$ einem späteren Behandlungsbeginn bei chronisch HIV-Infizierten und die bessere virologische Wirksamkeit der heute verwendeten Behandlungsregime. Trotz einer Zunahme von Resistenzmutationen bei antiretroviral Behandelten sinkt daher in den letzten Jahren das Risiko der Übertragung resistenter Varianten [Routy et al. Abstr. 678].

Eine Übertragung von resistenten Viren erfolgt in der Regel durch ungeschützte Sexualkontakte und Nadeltausch mit Patienten, die sich bereits in Behandlung befinden. In einer amerikanischen Studie wurden in Behandlung befindliche HIVPatienten zu ihrem sexuellen Risikoverhalten befragt. Eine Minderheit von 15-25\% berichtet über ungeschützte sexuelle Kontakte. Ein Zusammenhang zwischen ungeschütztem Sex und Viruslast oder Resistenzstatus konnte dabei nicht festgestellt werden. Über einen Zeitraum von $6 \mathrm{Mo-}$ naten wurden pro Patient mit resistentem Virus, der ungeschützte Sexualkontakte angab, zwischen einem und 2 Partner exponiert, meist mehrfach [Kozal et al. Abstr. 35LB].
HIV-HCV-Koinfektion

\section{Kommentar zur APRICOT-Studie, von Prof. Jürgen Rockstroh, Universitätsklinik Bonn}

Seit Einführung der hochaktiven antiretroviralen Therapie (HAART) sind HIVassoziierte Morbidität und Mortalität signifikant zurückgegangen. Parallel kam es jedoch zu einer deutlichen Zunahme an lebertypischen Komplikationen bei mit einer Hepatitis C koinfizierten HIV-Patienten. In der Tat weist gut ein Drittel aller europäischen HIV-Patienten zusätzlich zu ihrer HIV-Infektion gleichzeitig eine Hepatitis C auf. Im Rahmen der HIV-assoziierten Immundefizienz kommt es zu einem deutlich beschleunigten Verlauf der Hepatitis $\mathrm{C}$, und die Leberzirrhoserate liegt bei HIV/HCV-koinfizierten Patienten mehr als 5-mal höher als bei HCV-monoinfizierten Patienten. Unter Berücksichtigung der erheblichen klinischen Bedeutung der rascher verlaufenden Hepatitis-C-Koinfektion bei HIV-Patienten und der hierdurch verursachten Morbidität und Mortalität ist die Entwicklung von Therapiestrategien zur Behandlung der Hepatitis-C-Koinfektion bei HIV-Patienten von größter klinischer Bedeutung.

Im Rahmen des AIDS-Kongresses in San Francisco im Februar 2004 wurden erstmals Daten der APRICOT-Studie vorgestellt, die die bisher größte Studie zur Behandlung der HCV-Koinfektion bei HIVPatienten darstellt. Innerhalb dieser internationalen Studie wurden in 3 Armen pegInterferon- $\alpha 2 \mathrm{a}$ (Pegasys) \pm Ribavirin vs. Interferon- $\alpha 2 \mathrm{a}+$ Ribavirin zur Behandlung der Hepatitis C bei HIV/HCV-Koinfektion untersucht. Insgesamt konnten $880 \mathrm{~Pa}$ tienten aus 95 Zentren in 19 Ländern eingeschlossen werden. In die Studie wurden nur HIV/HCV-koinfizierte Patienten aufgenommen, die bislang keine Behandlung für ihre Hepatitis $\mathrm{C}$ erhalten hatten, nachweislich $\mathrm{HCV}$-seropositiv waren und eine quantifizierbare HCV-RNA aufwiesen. Zusätzlich mussten die Leberwerte erhöht sein und eine Leberbiopsie Zeichen einer Hepatitis-C-Infektion aufweisen. Patienten mit ausgeprägter Zirrhose konnten auch an dieser Untersuchung teilnehmen. Von Seiten der HIV-Infektion mussten entweder stabile Helferzellzahlen von
>200 Zellen/ $\mu$ l oder aber Helferzellen zwischen 100 und 200/ $\mathrm{Ll}$ bei niedriger $\mathrm{HI}-\mathrm{Vi}$ rämie von $<5.000 \mathrm{Kopien} / \mathrm{ml}$ vorliegen. $\mathrm{Pa}$ tienten mit stabiler HIV-Infektion konnten mit oder ohne antiretrovirale Behandlung in die Studie eingeschlossen werden. Über $80 \%$ der Patienten in allen 3 Armen waren männlich, das Durchschnittsalter lag bei 40 Jahren, der Anteil der Zirrhotiker lag in allen 3 Armen um die 15\%. Der überwiegende Teil der Patienten hatte sich die HCV-Infektion über i.v.-Drogenkonsum zugezogen (zwischen 62 und 71\%). Die HCV-RNA lag bei Studienbeginn in allen 3 Gruppen zwischen 5,2 und 6,3 IU/ $\mathrm{ml} \cdot 10^{6}$. Gut zwei Drittel der Patienten wiesen einen HCV-Genotypen 1 oder 4 auf. Über $84 \%$ der Patienten erhielten eine HIV-spezifische antiretrovirale Therapie bei darunter gut eingestellter HIV-Infektion mit einer durchschnittlichen Viruslast von 2,3 $\pm 1,0 \log _{10}$ Kopien/ml. 60\% der Patienten lagen mit ihrer HIV-RNA unterhalb von 50 Kopien, die mittlere Helferzellzahl lag in allen 3 Studienarmen zwischen 522 und $542 / \mu \mathrm{l}$.

24 Wochen nach Therapieende zeigte sich ein dauerhaftes virologisches Ansprechen (definiert als HCV-RNA unter der Nachweisgrenze) bei 40\% der mit Pegasys+Ribavirin behandelten Patienten. Dies war signifikant höher als bei den Patienten, die nur Pegasys+Placebo (20\%) oder Interferon- $\alpha 2 \mathrm{a}+$ Ribavirn (12\%) erhalten hatten. In einer weiter gehenden Subanalyse, bezogen auf das virologische Ansprechen in Abhängigkeit des vorliegenden Genotyps, zeigte sich für Genotyp 1 zum Ende der Behandlung ein Ansprechen von 38\% im peg-Interferon- $\alpha 2 a+$ Ribavirin-Arm und von $29 \% 24$ Wochen nach Therapieende. Deutlich günstigere Ansprechraten konnten für die Genotypen 2 und 3 mit 64\% Ansprechrate bei Therapieende und $62 \% 24$ Wochen nach Therapieende festgestellt werden. Damit sind die Ansprechraten aus der APRICOT-Studie deutlich höher als die Ergebnisse bislang veröffentlichter Studien, die allerdings deutlich kleinere Fallzahlen aufwiesen.

Im Rahmen des amerikanischen HIVKongresses wurden auch 2 weitere, etwas größere Behandlungsstudien vorgestellt, die insgesamt jedoch mit dem pegylierten Interferon+Ribavirin-Arm in der amerikanischen Studie nur ein anhaltendes Thera- 
pieansprechen von insgesamt $27 \%$, für Genotyp 1 gar nur von $14 \%$ erreichten. In der französischen Studie war ein anhaltendes virologisches Ansprechen in dem pegyliertes Interferon+Ribavirin-Arm 24 Wochen nach Therapieende bei nur 27\% der Patienten beobachtet worden, bei Genotyp 1 oder 4 lag die Ansprechrate sogar nur bei 11\%.

Wenn man die Ergebnisse der 3 Studien vergleicht, müssen für die Interpretation verschiedene wichtige Punkte festgehalten werden:

Die mit Abstand größte eingeschlossene Patientenzahl findet sich in der APRICOT-Studie, sodass sich unter Berücksichtigung des guten Ansprechens innerhalb dieser Studie im pegylierten Interferon- $\alpha 2 a+$ Ribavirin-Arm eine entsprechende Behandlungskombination bei allen koinfizierten Patienten mit einer Behandlungsindikation empfiehlt. Unter Berücksichtigung des schlechteren Abschneidens der amerikanischen Studie, bei der allerdings initial mit einer niedrigen Ribavirindosis von nur 600 mg/Tag begonnen wurde, scheint die Einhaltung der auch für die Monoinfektion empfohlenen Ribavirindosis (800 mg für Genotyp 2 und 3 und 1.000-1.200 mg in Abhängigkeit des Körpergewichts für Genotyp 1 und 4) empfehlenswert, da offensichtlich für das Abschneiden der Patienten auflange Sicht, insbesondere bei Genotyp 1 und 4, die Initialtherapie mit entscheidend $\mathrm{zu}$ sein scheint. Unter Berücksichtigung der ausgesprochen geringen Relapserate in der Patientengruppe mit Genotyp 2 und 3 innerhalb der APRICOT-Studie wird deutlich, dass offensichtlich eine längere Behandlungsdauer von 48 Wochen mit einem wesentlich günstigeren Therapieansprechen auf lange Sicht verbunden ist als unter der bei Monoinfektion üblichen kurzen Behandlungsdauer von nur 24 Wochen. Spanische und deutsche Untersuchungen bei HIV/HCV-koinfizierten $\mathrm{Pa}$ tienten mit bereits abgeschlossenen Studienergebnissen zeigten eine fast 50\%ige Relapserate bei Genotyp 2 und 3 nach einer Behandlungszeit von 24 Wochen, sodass ab sofort die längere Behandlungsdauer als Standard bei der Behandlung von HIV/HCV-koinfizierten Patienten anzusehen ist.

Die hohe Abbruchrate von fast $40 \%$ in beiden Studienarmen der französischen
Untersuchung deutet auf die Komplexität und Schwierigkeiten beim Management der Behandlung von HIV/HCV-koinfizierten Patienten hin. Hierbei scheint insbesondere die Auswahl des jeweiligen HAART-Regimes von größter Bedeutung. Die hohe Rate an Laktatazidosen und Pankreatitiden unter vornehmlich ddI enthaltenden Regimen im französischen Patientenkollektiv spricht hier eine deutliche Sprache. Die Kombination von Didanosin und Ribavirin gilt inzwischen als kontraindiziert.

Auch bei der Kombination von d4T und Ribavirin sollte größte Vorsicht walten, bei der Kombination von AZT und Ribavirin ist von einem verstärkten Auftreten von Anämien auszugehen. Beim amerikanischen HIV-Kongress vorgestellte Daten zur supportiven Gabe von Erythropoetin zur Vermeidung von hämatologischen Komplikationen zeigten beeindruckende positive Ergebnisse, sodass hier individuell bei erhöhtem Risiko oder Auftreten entsprechender Komplikationen über eine supportive Behandlung mit Erythropoetin nachzudenken ist.

Der Umstand, dass in allen 3 Studien die HCV-RNA-Bestimmung bei Woche 12 zuverlässig vorhersagt, ob eine weitere $\mathrm{Be}$ handlung erfolgreich ist, kristallisiert die Woche 12 als Entscheidungspunkt für das weitere Vorgehen innerhalb der Therapie heraus. Patienten, die bis Woche 12 nicht angesprochen haben (mindestens 2 logStufen Abfall in der HCV-RNA), können ihre HCV-Behandlung abbrechen.

Interessante Daten kommen auch aus der amerikanischen Studie, die bei den Patienten, die nicht angesprochen haben, dennoch bei gut der Hälfte der Patienten eine histologische Verbesserung aufzeigen konnten, sodass eine HCV-Behandlung auch bei Nichterreichen der Viruseradikation zumindest für den Gesamtverlauf, was Histologie und auch Auftreten von HCC angeht, möglicherweise eine positive Rolle einnimmt.

Unter Berücksichtigung der neuen Aspekte bei der Behandlung HIV/HCVkoinfizierter Patienten sind im Januarheft von AIDS die entsprechend überarbeiteten Richtlinien zur Betreuung von Patienten mit HIV und Hepatitis-C-Koinfektion veröffentlicht worden (AIDS 2004; 18:1-12).
Dieser Bericht bezieht sich größtenteils auf Konferenzabstracts, die auf der offiziellen Webseite der Konferenz unter http://www.retroconference.org/2004/AbstractSearch/AbstractSearch.aspx auffindbar sind.

\section{Referenzen}

Darüber hinaus wurde folgende Publikation zur Erstellung dieses Berichtes herangezogen:

Martinez E, Arnaiz JA, Podzamczer D et al. (2003) Substitution of nevirapine, efavirenz, or abacavir for protease inhibitors in patients with human immunodeficiency virus infection. N Eng J Med 349:10361046

\section{Korrespondierender Autor Dr. U. Marcus}

Robert Koch-Institut, Nordufer 20, 13353 Berlin E-Mail: marcusu@rki.de 